\title{
A new experimental approach is required in the molecular analysis of intestinal neuronal dysplasia type $B$ patients
}

\author{
AVENCIA SÁNCHEZ-MEJÍAS ${ }^{1,2}$, RAQUEL M. FERNÁNDEZ ${ }^{1,2}$, \\ GUILLERMO ANTIÑOLO ${ }^{1,2}$ and SALUD BORREGO ${ }^{1,2}$ \\ ${ }^{1}$ Unidad de Gestión Clínica de Genética, Reproducción y Medicina Fetal, Instituto de Biomedicina de Sevilla (IBIS), \\ Hospitales Universitarios Virgen del Rocío/CSIC/Universidad de Sevilla; \\ ${ }^{2}$ Centro de Investigación Biomédica en Red de Enfermedades Raras (CIBERER), Sevilla, Spain
}

Received June 8, 2010; Accepted July 29, 2010

DOI: $10.3892 /$ etm. 2010.140

\begin{abstract}
Intestinal neuronal dysplasia type B (INDB) is characterized by the malformation of the parasympathetic submucous plexus of the gut. It is generally accepted that INDB has a genetic basis, and several genes produce an INDB-like phenotype in mice when disrupted, such as EDNRB. However, no mutations associated with this disease have been identified in several series analysed. In the present studu, we sought to determine whether the EDNRB/EDN3 signalling pathway plays a role in the pathogenesis of INDB in humans. Denaturing high performance liquid chromatography (dHPLC) techniques were employed to screen the $E D N R B$ and $E D N 3$ coding regions in 23 INDB patients. In addition, association studies were performed on these genes with single nucleotide polymorphisms strategically selected and genotyped by TaqMan technology. Although several novel variants were detected in both genes, none of these variants appeared to play a functional role in protein function or expression. Our results indicate that additional screening of other candidate genes in larger patient series is required to elucidate the molecular basis of INDB. Additionally, the systematic lack of positive results in the screening of candidate genes for INDB reported in the literature, together with our results, leads us to propose that INDB may alternatively arise as a consequence of gain of function mutations in genes related to enteric nervous system development. Therefore, the use of different molecular approaches, such as screening for genetic duplication or enhancer mutations, is recommended for future studies on the genetic basis of INDB.
\end{abstract}

Correspondence to: Dr Salud Borrego, Unidad de Gestión Clínica de Genética, Reproducción y Medicina Fetal, Hospitales Universitarios Virgen del Rocío, Avda. Manuel Siurot s/n, 41013 Sevilla, Spain

E-mail: salud.borrego.sspa@juntadeandalucia.es

Key words: intestinal neuronal dysplasia type B, Hirschsprung disease, enteric nervous system disorders, $E D N 3, E D N R B$

\section{Introduction}

Intestinal neuronal dysplasia type B (INDB; OMIM 601223) is a malformation of the enteric nervous system (ENS) that accounts for over $95 \%$ of cases of isolated IND (1) and is characterized by malformation of the submucosal plexus. Children with INDB present with intractable constipation and grossly slowed intestinal transit time. The histological features of INDB include hyperplasia of the submucosal plexus, giant ganglia, ectopic ganglion cells at the muscular and mucosa layers, and increased acetylcholinesterase activity in the lamina propria and around submucosal blood vessels, which indicates immaturity of the ENS (1). The clinical picture of INDB resembles Hirschsprung disease (HSCR; OMIM 142623), a congenital disorder characterized by the absence of intramural ganglion cells in the myenteric and submucosal plexuses along a variable portion of the distal intestine. INDB does not include a region of aganglionosis, in absolute contrast to HSCR, but as in HSCR, it is reported to sometimes show increased extrinsic nerve fibers in the affected gut (2). In addition, some investigators have reported that $25-35 \%$ of patients with HSCR have associated INDB (2). The lack of unified criteria for the diagnosis of INDB has led to doubt regarding whether INDB exists as a distinct histopathology entity. Moreover, the clinical features of INDB are shared in common with immaturity of the ENS and arise in healthy individuals at an early age. However, a concerted effort has been made by scientists and clinicians to establish some generally accepted criteria for the diagnosis of INDB (2). This has led to great advances in the understanding of its pathological basis.

The etiopathogenesis of INDB remains obscure. It is generally accepted that it is caused by a delay in ENS maturation, although its association with intestinal chronic obstruction and HSCR indicates that it may arise as a secondary response to obstruction or inflammation of the bowel, either in the foetal or postnatal period (3). The genetic basis and congenital origin of INDB were determined based on a study of affected monozygotic twins and on reports of families in which several members had biopsy-proven INDB across multiple generations, although with no specific identified genetic alterations $(4,5)$. Several genes have been described to play some aetiological role in HSCR pathogenesis, usually related to the developmental 
Table I. Sequence variants detected in $E D N R B$ and $E D N 3$ mutational screening in INDB patients.

\begin{tabular}{|c|c|c|c|c|}
\hline Gene & $\begin{array}{l}\text { Nucleotide } \\
\text { change }\end{array}$ & $\begin{array}{l}\text { Amino acid } \\
\text { change }\end{array}$ & $\begin{array}{c}\text { Novel/previously } \\
\text { described }\end{array}$ & $\begin{array}{l}\text { Allelic frequency in } \\
\text { control population }(\%)\end{array}$ \\
\hline
\end{tabular}

EDNRB

c. $732 \mathrm{G}>\mathrm{A}$
c. $802-139 \mathrm{~A}>\mathrm{G}$
c. $802-122 \mathrm{~T}>\mathrm{C}$
c. $831 \mathrm{~A}>\mathrm{G}$
c. $914 \mathrm{G}>\mathrm{A}$

L277L

S305N

$\begin{array}{cr}\text { Novel } & 0.5 \\ \text { Novel } & 0.0 \\ \text { rs9530703 } & 6.7 \\ \text { rs5351 } & 56.5 \\ \text { rs5352 } & 2.3\end{array}$

EDN3

$$
\begin{aligned}
& \text { c. } 53-57 \mathrm{C}>\mathrm{T} \\
& \text { c. } 365+23 \mathrm{G}>\mathrm{A} \\
& \text { c. } 621-80 \mathrm{~T}>\mathrm{C} \\
& \text { c. } 240 \text { delCC }
\end{aligned}
$$

$\begin{array}{cr}\text { Novel } & 4.0 \\ \text { rs } 11570257 & 4.0 \\ \text { rs11570349 } & 0.0 \\ \text { rs34516274 } & 28.5\end{array}$

programme of neural crest cells (6). Since HSCR and INDB are ENS disorders that frequently occur in combination, common molecular pathways involved in the genesis of the two pathologies may exist. However, no mutations have been found in the most relevant genes implicated in HSCR, such as RET and $G D N F$, in several INDB patient series $(7,8)$, or in other screened genes $(5,9,10)$. By contrast, a certain combination of single nucleotide polymorphisms (SNPs) in the RET protooncogene was found to be associated with the INDB phenotype in a previous study performed by our group (8).

The mutation spotting lethal (sl) at Ednrb which, in homozigosis, leads to aganglionosis in rats, provokes, in heterozygosis, hyperganglionosis and giant ganglia in the submucosal plexus (11). In addition, giant ganglia have also been observed in the aganglionic region of $E d n 3$-deficient mice (12). The EDNRB and $E D N 3$ genes have previously been evaluated as susceptibility genes for INDB, with no mutations identified (7). However, the sensibility of the technique used was not sufficient to completely exclude these genes as INDB susceptibility factors. In addition, the absence of coding mutations in these genes does not rule out the existence of other variants that may confer susceptibility to INDB, as is the case with the RET high-susceptibility-risk haplotype for HSCR (13). The aim of the present study was to determine whether the EDNRB/EDN3 signalling pathway plays a role in the pathogenesis of INDB. To this end, a mutational screening of its coding sequence was conducted, and the identified polymorphisms and haplotypes were evaluated as susceptibility factors for this disease.

\section{Patients and methods}

The study comprised a total of 23 patients presenting with INDB histologically diagnosed using the criteria updated by Meier-Ruge et al (2). Of note, one of the INDB patients included in this series had a monozygotic twin, also affected by INDB.

Triads composed of the patient and both parents were complete for 22 of the patients. In addition, a control group comprising 150 unrelated, race, age and gender-matched individuals, without any symptoms suggestive of ENS alterations, was analyzed. Informed consent was obtained from all the participants for clinical and molecular genetic studies. The study conformed to the tenets of the declaration of Helsinki and was approved by the Hospitales Universitarios Virgen del Rocío IRB. Mutational screening was carried out by denaturing high performance liquid chromatography (dHPLC) and sequence analysis, as previously described (14).

Large-scale genotyping of EDNRB and END3 polymorphisms was performed in the INDB triads and controls as previously described (14). Allelic, genotypic and haplotypic frequencies and distributions were compared between patients and controls by SPSS version 15.0 for Windows. In addition, previously obtained data from 196 isolated HSCR patients were used (14). In each analysis, statistical significance was calculated using Pearson's $\chi^{2}$ test, with statistical significance set at $\alpha=0.05$. Haplotypes comprising the EDNRB and EDN3 polymorphisms analysed were generated based on the results of the complete triads when available (patient, father and mother), which allowed us to reconstruct and compare the transmitted vs. non-transmitted alleles. With these results, we proceeded to compare the distribution of haplotypes among the patients and controls. Statistical estimates were calculated using the method outlined above.

\section{Results}

The mutational screening of the EDNRB and EDN3 coding regions in 23 INDB patients revealed the presence of nine sequence variants, three of which had never been previously reported (Table I). Since both the silent changes and intronic variants generate no variation at the protein sequence level, it was more probable that their pathogenic mechanism, if any, would operate by affecting transcript stability, RNA splicing or DNA-protein binding. Thus, as a first approach, those variants were submitted to several splice site and transcription factor binding site sequence prediction interfaces (http://www.fruitfly. org/seq_tools/splice.html; http://www.fruitfly.org/cgi-bin/seq_ tools/promoter.pl; http://www.ebi.ac.uk/asd-srv/wb.cgi). None of these variants were predicted to have any impact on the expression and maturation of mRNA in silico.

The most notable result was a heterozygous conservative substitution of serine to asparagine at codon 305 of the 
Table II. Allelic distribution and frequency of EDNRB and EDN3 genotyped variants among patient and control groups.

\begin{tabular}{|c|c|c|}
\hline Variant & INDB vs. HSCR (\%) & INDB vs. controls (\%) \\
\hline \multicolumn{3}{|c|}{$E D N R B$} \\
\hline \multicolumn{3}{|c|}{ c. $484-4125 \mathrm{C}>\mathrm{T}$} \\
\hline $\mathrm{C}$ & $37(80.4)$ vs. $186(84.5)$ & $37(80.4)$ vs. $170(83.3)$ \\
\hline \multirow[t]{2}{*}{$\mathrm{T}$} & 9 (19.6) vs. 34 (15.5) & 9 (19.6) vs. 34 (16.7) \\
\hline & $\chi^{2}=0.40, p=0.5266483$ & $\chi^{2}=0.22, p=0.63794669$ \\
\hline \multicolumn{3}{|l|}{ I187I } \\
\hline $\mathrm{C}$ & 46 (100) vs. 219 (99.5) & 46 (100) vs. 202 (100) \\
\hline \multirow[t]{2}{*}{$\mathrm{T}$} & $0(0)$ vs. $1(0.5)$ & $0(0)$ vs. $0(0)$ \\
\hline & Fisher's $\mathrm{p}=1$ & NA \\
\hline \multicolumn{3}{|c|}{ c. $+1985 \mathrm{G}>\mathrm{A}$} \\
\hline $\mathrm{G}$ & 35 (87.5) vs. 188 (88.7) & 35 (87.5) vs. $172(86.0)$ \\
\hline \multirow[t]{2}{*}{ A } & 5 (12.5) vs. 24 (11.3) & 5 (12.5) vs. $28(14.0)$ \\
\hline & $\chi^{2}=0.05, p=0.8302636$ & $\chi^{2}=0.06, p=0.8014447$ \\
\hline \multicolumn{3}{|l|}{ EDN3 } \\
\hline \multicolumn{3}{|c|}{ c. $365+7474 \mathrm{~T}>\mathrm{C}$} \\
\hline $\mathrm{T}$ & $32(66.7)$ vs. $154(80.5)$ & 32 (66.7) vs. 127 (63.5) \\
\hline \multirow[t]{2}{*}{$\mathrm{C}$} & 16 (33.3) vs. 64 (19.5) & $16(33.3)$ vs. $73(36.5)$ \\
\hline & $\chi^{2}=0.30, p=0.5866268$ & $\chi^{2}=0.63, p=0.4262079$ \\
\hline \multicolumn{3}{|c|}{ c. $366-3935 \mathrm{C}>\mathrm{G}$} \\
\hline $\mathrm{C}$ & 37 (80.4) vs. 182 (75.9) & 37 (80.4) vs. $176(83.8)$ \\
\hline \multirow[t]{2}{*}{ G } & 9 (19.6) vs. 38 (24.1) & 9 (19.6) vs. 34 (16.2) \\
\hline & $\chi^{2}=0.14, p=0.7108324$ & $\chi^{2}=0.31, p=0.5792162$ \\
\hline \multicolumn{3}{|c|}{ c. ${ }^{*} 231+236 \mathrm{G}>\mathrm{A}$} \\
\hline G & 32 (69.6) vs. 169 (76.8) & 32 (69.6) vs. 156 (83.9) \\
\hline \multirow[t]{2}{*}{ A } & 14 (30.4) vs. 51 (23.2) & $14(30.4)$ vs. $30(16.1)$ \\
\hline & $\chi^{2}=1.08, p=0.2978297$ & $\chi^{2}=2.16, p=0.1415837$ \\
\hline
\end{tabular}

EDNRB gene in two independent patients. This variant was previously described in an HSCR patient and was proposed to be a causative mutation due to the hypothetical disruption of a putative phosphorylation site and the absence of the variant in 50 control individuals tested (15). However, in the present study, we detected this variant in 5 out of 150 control individuals in a heterozygous state. These results are in accordance with the available data on the genotypic frequency of S305N in a European population (http://www.ncbi.nlm.nih.gov/SNP). Thus, it seems that the $\mathrm{S} 305 \mathrm{~N}$ variant is a rare polymorphism rather than a mutation related to HSCR or INDB.

The genotypic data of three EDNRB SNPs (IVS1$4125 \mathrm{C}>\mathrm{T}, \mathrm{c} .561 \mathrm{C}>\mathrm{T}$ and c.*1985 G>A) and three EDN3 SNPs (IVS2+7474T, IVS2-3935 C>G and IVS5+236 G>A) in the INDB patients and controls were obtained using TaqMan technology. These data, together with the available data from 196 HSCR patients previously obtained by our group (14), were used to analyse the allelic (Table II) and genotypic (Table III) distributions among the patient and control groups. No statistical differences were found in the distribution of any of these variants or the generated haplotypes comprising them when INDB patients were compared to HSCR patients or when INDB patients were compared to control individuals. Additionally, no statistical differences were obtained in a transmission disequilibrium test (TDT) analysis (data not shown).

\section{Discussion}

The pathogenesis of INDB is not completely understood and its aetiology remains unknown, although several pieces of evidence suggest that it has a genetic basis. The presence of histological features characteristic of INDB in murine models with heterozygous mutations at Ednrb and Edn3 $(11,12)$ indicate that this signalling pathway may be implicated in the pathogenesis of INDB, as is the case in HSCR. However, the results presented here and in previous reports (7) fail to reveal any causal variant in IDNB patients. We also failed to find any association between SNPs or the haplotypes comprising them and the disease. The lack of associations between INDB and the $E D N R B$ or $E D N 3$ genes suggests that the endothelin signalling pathway is not implicated in the pathogenesis of this disease. However, the small patient sample presented here does not allow this hypothesis to be completely ruled out. In fact, a low number of patients is a recurring problem in the analysis of the genetic basis of IDNB $(5,7,9,10)$, due to the low prevalence of the disease and the difficulties involved in the clinical diagnosis and management of patients.

Several animal models with mutations of various genes involved in ENS development, such as Hox1L11, Spry2, Ednrb and $E d n 3$, share histological features with the INDB phenotype in humans $(11,16-18)$, suggesting that hyperganglionosis 
Table III. Genotypic distribution and frequency of EDNRB and EDN3 genotyped variants among patient and control groups.

\begin{tabular}{|c|c|c|}
\hline Variant & DNI B vs. HSCR (\%) & DNI B vs. controls (\%) \\
\hline \multicolumn{3}{|c|}{$E D N R B$} \\
\hline \multicolumn{3}{|c|}{ c. $484-4125 \mathrm{C}>\mathrm{T}$} \\
\hline $\mathrm{CC}$ & 16 (69.6) vs. 79 (71.2) & 16 (69.6) vs. 70 (69.6) \\
\hline $\mathrm{CT}$ & 5 (21.7) vs. $29(26.1)$ & 5 (21.7) vs. 30 (29.4) \\
\hline \multirow[t]{2}{*}{$\mathrm{T}$} & 2 (8.7) vs. 3 (2.7) & 2 (8.7) vs. $2(2.0)$ \\
\hline & $\chi^{2}=0.08, p=0.7721618$ & $\chi^{2}=0.32, p=0.5695065$ \\
\hline \multicolumn{3}{|l|}{ I187I } \\
\hline $\mathrm{CC}$ & 23 (100) vs. 109 (99.1) & 23 (100) vs. $101(100)$ \\
\hline $\mathrm{CT}$ & 0 (0) vs. $1(0.9)$ & 0 (0) vs. $0(0.0)$ \\
\hline \multirow[t]{2}{*}{$\mathrm{T}$} & $0(0)$ vs. $0(0.0)$ & $0(0)$ vs. $0(0.0)$ \\
\hline & Fisher's $\mathrm{p}=1$ & NA \\
\hline \multicolumn{3}{|c|}{ c. $+1985 \mathrm{G}>\mathrm{A}$} \\
\hline GG & 15 (75.5) vs. 82 (87.4) & 15 (75.5) vs. $72(72.0)$ \\
\hline GA & 5 (25.5) vs. $24(22.6)$ & 5 (25.5) vs. $28(28.0)$ \\
\hline \multirow[t]{2}{*}{ AA } & $0(0)$ vs. $0(0.0)$ & $0(0)$ vs. $0(0.0)$ \\
\hline & $\chi^{2}=0.05, p=0.8302636$ & $\chi^{2}=0.08, p=0.7838616$ \\
\hline \multicolumn{3}{|c|}{ EDN3 } \\
\hline \multicolumn{3}{|c|}{ c. $365+7474 \mathrm{~T}>\mathrm{C}$} \\
\hline TT & 10 (41.7) vs. $60(55.0)$ & $10(41.7)$ vs. $42(40.0)$ \\
\hline $\mathrm{TC}$ & $12(50)$ vs. $34(31.2)$ & $12(50)$ vs. 43 (41.0) \\
\hline \multirow[t]{2}{*}{$\mathrm{CC}$} & 2 (8.3) vs. 15 (13.8) & 2 (8.3) vs. $20(19.0)$ \\
\hline & $\chi^{2}=3.13, p=0.20867686$ & $\chi^{2}=1.70, p=0.42667582$ \\
\hline \multicolumn{3}{|c|}{ c. $366-3935 \mathrm{C}>\mathrm{G}$} \\
\hline $\mathrm{CC}$ & 14 (60.9) vs. 75 (68.2) & $14(60.9)$ vs. $75(60.0)$ \\
\hline CG & 9 (39.1) vs. $32(29.1)$ & 9 (39.1) vs. 46 (36.8) \\
\hline \multirow[t]{2}{*}{ GG } & $0(0)$ vs. $3(2.7)$ & 0 (0) vs. $4(3.2)$ \\
\hline & $\chi^{2}=1.40, p=0.49628003$ & $\chi^{2}=0.01, p=0.9197185$ \\
\hline \multicolumn{3}{|c|}{ c. $* 231+236 \mathrm{G}>\mathrm{A}$} \\
\hline GG & 11 (47.8) vs. 66 (60.0) & $11(47.8)$ vs. 61 (62.2) \\
\hline GA & $10(43.5)$ vs. 37 (33.6) & $10(43.5)$ vs. $34(34.7)$ \\
\hline \multirow[t]{2}{*}{$\mathrm{AA}$} & 2 (8.7) vs. $7(6.4)$ & 2 (8.7) vs. 3 (3.1) \\
\hline & $\chi^{2}=1.16, p=0.55955014$ & $\chi^{2}=1.02, p=0.3119877$ \\
\hline
\end{tabular}

in ENS could be due to defects in a variety of such genes, alone or in combination. For this reason, it would be useful to completely characterize the genetic background of patients with INDB and to analyse whether different genes interact in order to increase the number of nerve cells and ganglia. Given the negative results obtained to date in studies of the molecular causes of INDB $(5,7,9,10)$, we suggest the adoption of a completely new experimental approach to elucidate the genetic basis of INDB. In this vein, animal models with deleted Bmp4, a protein implicated in ENS formation, present hypoganglionosis (19). It is tempting to speculate that the overexpression of BMP4 protein may cause an increase in the number and size of enteric ganglia. In fact, overexpression of $\mathrm{Ntf} 3$ promotes hyperplasia of the myenteric plexus in rats (20). In the same manner, we propose that INDB arises as a consequence of gain of function mutations in genes related to ENS development. Therefore, a different molecular approach to studying the genetic basis of INDB, which includes screening for genetic duplications or enhancer mutations, may be required.

Although the genetic basis of INBD remains to be elucidated, it must be acknowledged that our results and those presented elsewhere $(5,7,9,10)$ also support the possibility that INDB is a secondary consequence of environmental factors, such as intestinal stenosis or intestinal obstruction (21). It is well known that individuals are not only a result of their genetic information, but also of interaction with their surrounding environment. Despite the importance that intestinal obstructive lesions may play in the INDB phenotype, we believe that, before ruling out a genetic basis, it is necessary to completely analyse the genetic factors that may be associated with this disease by evaluating an adequate number of patients and by adopting alternative experimental approaches.

\section{Acknowledgements}

We would like to thank all the patients who participated in the study, as well as their families for their collaboration. This study was funded by the Fondo de Investigación Sanitaria, Instituto de Salud Carlos III (ISCIII), Spain (PI070080, and PI071315 for the E-Rare project), Consejería de Salud (PI0249-2008) de la Junta de Andalucía, Spain; and Consejería de Innovación Ciencia y Empresa (CTS-2590) de la Junta de 
Andalucía, Spain. The CIBER de Enfermedades Raras is an initiative of ISCIII. A.S.M. is a pre-doctoral fellow funded by ISCIII, Spain.

\section{References}

1. Puri P and Shinkai T: Pathogenesis of Hirschsprung's disease and its variants: recent progress. Semin Pediatr Surg 13: 18-24, 2004.

2. Meier-Ruge WA, Bruder E and Kapur RP: Intestinal neuronal dysplasia type B: one giant ganglion is not good enough. Pediatr Dev Pathol 9: 444-452, 2006.

3. Galvez Y, Skaba R, Vajtrova R, Frantlova M and Herget J: Evidences of secondary neuronal intestinal displasia in a rat model of chronic intestinal obstruction. J Invest Surg 17: 31-39, 2004.

4. Kobayashi H, Mahomed A and Ouri P: Intestinal neuronal dysplasia in twins. JPGN 22: 398-401, 1996.

5. Costa M, Fava M, Seri M, et al: Evaluation of the HOX11L1 gene as a candidate for congenital disorders of intestinal innervation. J Med Genet 37: E9, 2000.

6. Amiel J, Sproat-Emison E, Garcia-Barcelo M, et al: Hirschsprung Disease Consortium. Hirschsprung disease, associated syndromes and genetics: a review. J Med Genet 45: 1-14, 2008.

7. Gath R, Goessling A, Keller K-M, et al: Analysis of the RET, GDNF, EDN3 and EDNRB genes in patient with intestinal neuronal dysplasia and Hirschprung disease. Gut 48: 671-675, 2001.

8. Fernandez RM, Sanchez-Mejias A, Ruiz-Ferrer M, Lopez-Alonso M, Antiñolo G and Borrego S: Is the RET protooncogene involved in the pathogenesis of intestinal neuronal displasia type B? Mol Med Rep 2: 265-270, 2009.

9. Fava M, Borghini S, Cinti R, et al: HOX11L1: a promoter study to evaluate possible expression defects in intestinal motility disorders. Int J Mol Med 10: 101-106, 2002.

10. Borghini S, Duca MD, Pini Prato A, et al: Search for pathogenetic variants of the SPRY2 gene in intestinal innervation defects. Intern Med J 39: 335-337, 2009.
11. Von Boyen GB, Krammer HJ, Suss A, Dembowski C, Ehrenreich $\mathrm{H}$ and Wedel T: Abnormalities of the enteric nervous system in heterozygous endothelin B receptor deficient (spotting lethal) rats resembling intestinal neuronal dysplasia. Gut 51: 414-419, 2002.

12. Sandgren K, Larsson LT and Ekblad E: Widespread changes in neurotransmitter expression and number of enteric neurons and interstitial cells od Cajal in lethal spotted mice. Dig Dis Sci 47: 1049-1064, 2002

13. Fernandez RM, Boru G, Peciña A, et al: Ancestral RET haplotype associated with Hirschsprung's disease shows linkage disequilibrium breakpoint at -1249. J Med Genet 42: 322-327, 2005.

14. Sánchez-Mejías A, Fernández RM, López-Alonso M, Antiñolo G and Borrego S: New roles of EDNRB and EDN3 in the pathogenesis of Hirschsprung disease. Genet Med 12: 39-43, 2010.

15. Auricchio A, Casari G, Staiano A and Ballabio A: Endothelin-B receptor mutations in patients with isolated Hirschsprung disease from a non-inbred population. Hum Mol Genet 5: 351-354, 1996.

16. Yamataka A, Hatano M, Kobayashi $H$, et al: Intestinal neuronal dysplasia-like pathology in Ncx/Hox11L.1 gene-deficient mice. J Pediatr Sur 36: 1293-1296, 2001.

17. Yanai T, Kobayashi H, Yamataka A, et al: Acetylcholine-related bowel dysmotility in homozygous mutant NCX/HOX11L.1deficiented in causing intestinal neuronal dysplasia. J Pediatr Sur 39: 927-930, 2004.

18. Taketomi T, Yoshiga D, Taniguchi K, et al: Loss of mammalian Sprouty2 leads to enteric neuronal hyperplasia and esophageal achalasia. Nat Neurosci 8: 855-857, 2005.

19. Goldstein AM, Brewer KC, Doyle AM, Nandor $\mathrm{N}$ and Roberts DJ: BMP signaling is necessary for neural crest cell migration and genglion formation in the enteric nervous system. Mech Dev 122: 821-833, 2005.

20. Chalazonitis A, D’Autréaux F, Guha U, et al: Bone morphogenetic protein-2 and -4 limit the number of enteric neurons but promote development of a TrkC-expressing neurotrophin-3dependent subset. J Neurosci 24: 4266-4282, 2004.

21. Kapur RP: Neuronal dysplasia: a controversial pathological correlate of intestinal pseudo-obstruction. Am J Med Genet 122A: 287-293, 2003. 